\title{
Biologie de la mucoviscidose : progrès récents et perspectives
}

Le CFTR, protéine codée par le gène de la mucoviscidose, est un canal à chlore stimulé par l'AMP cyclique. Plus de cent mutations du gène ont été à ce jour détectées chez des malades atteints de mucoviscidose, la plus fréquente d'entre elles (la délétion de la phénylalanine 508) représentant $70 \%$ des cas, d'autres de 2 à $5 \%$, le plus grand nombre n'ayant été détectées que dans des cas isolés. La plus grande partie des mutations est regroupée dans les régions de la protéine fixant les nucléotides ou dans les domaines transmembranaires. Les conséquences pathologiques de ces mutations pourraient être non seulement la conséquence de l'insuffisance du fonctionnement du canal chlore, mais aussi d'un éventuel effet toxique de certaines protéines mutantes. Les mutations non-sens, censées ne permettre la synthèse d'aucune protéine-canal, semblent en effet avoir des conséquences moins graves que des mutations aboutissant à l'accumulation de protéines anormales.

\section{Michel Goossens}

\section{ADRESSE}

M. Goossens : professeur à la faculté de médecine. Laboratoire de génétique moléculaire, Inserm U. 91, hôpital Henri-Mondor, 94010

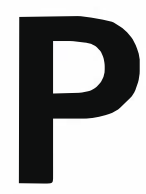

lus d'un an et demi après la découverte du gène de la mucoviscidose (fibrose kystique du pancréas, cystic fibrosis ou CF), la fonction de la protéine CFTR (cystic fibrosis transmembrane conductance regulator) reste à comprendre malgré les progrès notables effectués ces derniers mois. Rappelons que la mucoviscidose, la plus fréquente des maladies héréditaires rencontrées dans les populations européennes, est caractérisée par des anomalies du transport de l'eau et des électrolytes à travers les membranes cellulaires de plusieurs organes comme les poumons, les glandes sudoripares, l'intestin et le pancréas, et cliniquement par une atteinte pulmonaire et pancréatique. La perméabilité au chlorure $\left(\mathrm{Cl}^{-}\right)$de la membrane apicale des cellules épithéliales des voies aériennes supérieures est contrôlée par une famille de canaux protéiques dont l'ouverture est favorisée par des stimuli divers comme la concentration des ions $\mathrm{Ca}^{2}+$ ou l'augmentation de l'AMP cyclique intracellulaire. Ce dernier active une protéine kinase stimulée par l'AMP cyclique (PKA) qui pourrait phosphoryler un canal chlorure spécifique ou une protéine de régulation associée. Chez les malades, les canaux $\mathrm{Cl}^{-}$des membranes cellulaires des voies respiratoires sont présents mais ne peuvent être activés par l'AMP cyclique ou la protéine kinase. Cela a pour effet d'entraîner un blocage de la sécrétion de chlorures et d'eau vers la lumière de l'arbre respiratoire et conduit à l'épaississement du mucus bronchique, aux inf ections et à la maladie pulmonaire qui assombrit le pronostic vital de ces malades. L'isolement du gène de la mucoviscidose [1] a été le point de départ d'un ensemble de recherches dont les objectifs immédiats sont de comprendre le rôle de la protéine codée. L'analyse des mutations qui altèrent sa fonction, au-delà de leur simple inventaire, devrait à cet égard apporter des infor- 
mations utiles. Ce gène, dont l'analyse génétique a démontré la causalité, s'étend sur $250 \mathrm{~kb}$ d'ADN sur le chromosome 7 et code pour une protéine de 1480 acides aminés (CFTR). La mutation la plus fréquente est une délétion de trois nucléotides qui spécifient la phénylalanine $(\Delta \mathrm{F} 508)$. Elle constitue à elle seule $70 \%$ des allèles $\mathrm{CF}$, les autres étant caractérisés par (à ce jour) plus d'une centaine de mutations rares. Des travaux très récents viennent de confirmer les résultats de l'analyse génétique en démontrant que le défaut moléculaire pouvait être corrigé in vitro lorsque le gène CFTR normal est introduit dans des cellules épithéliales mutantes. Sur ces résultats peuvent maintenant s'appuyer les recherches nécessaires à la mise en œuvre de voies thérapeutiques nouvelles, pharmacologiques ou génétiques.

\section{Les mutations du gène $C F$}

- Une grande hétérogénéité moléculaire

A l'initiative des découvreurs du gène, les laboratoires engagés dans la caractérisation des mutations ont rapidement maillé un réseau de communication par télécopie (au sein d'un "Consortium international d'analyse génétique de la mucoviscidose ") dont le centre se trouve à Toronto, dans le laboratoire de Lap-Chee Tsui. Depuis deux ans, chaque mutation nouvelle caractérisée a été signalée à l'ensemble des membres actifs cooptés qui ont ainsi eu la possibilité de la rechercher et d'en déterminer la fréquence dans leur population de patients. Après plus d'un an de fonctionnement, le bilan est très satisfaisant: l'analyse des 27 exons du gène a conduit à la caractérisation de plus de 100 mutations. A côté de la délétion $\Delta \mathrm{F} 508$, quelques mutations ponctuelles sont retrouvées avec une fréquence non négligeable (2-5\% des mutations non- $\Delta$ F508), alors que les autres sont rares $(<1 \%)$. Les analyses génétiques en cours pourront, dans certains cas, renseigner sur l'origine de certaines d'entre elles (récurrence, effet fondateur) et leur répartition dans les populations. Certaines mutations rares sont en effet particulières à certains groupes ethniques: par exemple, la G551D* est retrouvée avec une fré$m / s n^{\circ} 10$, vol. 7 , décembre 91 quence plus élevée chez les Celtes (Bretagne, Royaume-Uni) ou dans le nord de l'Europe. Plusieurs mutations n'ont été identifiées jusqu'ici que chez un ou deux individus seulement. Il faut noter que l'effet délétère d'un certain nombre de substitutions "fauxsens " reste à démontrer par un test fonctionnel difficile à mettre en œuvre. Cette hétérogénéité rend pour l'instant impossible la mise en place d'un dépistage général, à grande échelle, qui semble souhaité par certains et dont la mise en œuvre éventuelle a fait, outre-Atlantique, l'objet d'un débat nourri. Dans le meilleur des cas, en effet, seulement 80 à $85 \%$ des anomalies peuvent être détectées: pour la population française, $70 \%$ des $\Delta$ F508 et deux ou trois autres mutations qui chacune constituent 2-5\% de l'ensemble (G551D, G542X**, $\left.\mathrm{W} 1282 \mathrm{X}^{* * *}\right)$, parfois plus selon les régions. Cette variété complique donc grandement le travail des laboratoires qui ont en charge leur diagnostic, car si la détection de la délétion $\Delta \mathrm{F} 508$ est aisée, elle ne permet d'identifier que $50 \%$ des couples à risque, ce qui donne, compte tenu des possibilités présentes d'analyse directe du gène, encore de beaux jours aux approches conventionnelles de détection du gène anormal fondées sur les analyses de liaison génétique.

Les corrélations génotypes/phénotypes semblent indiquer, au premier abord, que certaines mutations, notamment celles qui affectent les domaines hydrophobes ou transmembranaires, produisent une maladie de gravité moindre, comparées à d'autres, notamment à la $\Delta \mathrm{F} 508$ que l'on retrouverait pratiquement toujours associée à l'insuffisance pancréatique (une observation initiale qui ne semble pas vérifiée par tous les auteurs) et à l'atteinte pulmonaire grave. Quelques patients présentant une atteinte pancréatique sévère ont, de façon inattendue, une maladie pulmonaire d'expression modérée alors que les mutations non-sens qu'ils portent devraient entraîner, sinon une absence totale de CFTR, au minimum une amputation notable de la protéine [ 2 , 3]. Curieusement, un faisceau d'argu-

$$
\begin{aligned}
& { }^{*} \text { Gly } 551 \rightarrow \text { Asp. } \\
& { }^{* *} \text { Gly } 542 \rightarrow \text { stop. } \\
& \text { * }_{\text {Try } 1282} \rightarrow \text { stop. }
\end{aligned}
$$

ments convergents invite à suspecter que l'absence totale de CFTR fonctionnel pourrait être moins délétère que la présence d'un CFTR altéré par des mutations faux-sens ou de petites délétions, comme si d'autres facteurs pouvaient suppléer la fonction de cette molécule. Dans cette hypothèse, peuton imaginer que des sujets porteurs d'une délétion complète ou partielle du CFTR aient pu passer inaperçus puisque aucune délétion importante n'a encore été mise en évidence ? Une autre hypothèse à tester est celle d'un épissage alternatif de l'ARN messager, qui produirait des isoformes propres à certains tissus. Il est intéressant de savoir que des produits d'épissage alternatif ont été caractérisés dans les tissus normaux, le plus abondant étant amputé de l'exon 9. L'existence d'une protéine tronquée pouvant néanmoins s'intégrer dans la membrane et fonctionner dans certains tissus n'est pas non plus à exclure dans l'état actuel des connaissances.

Comme on le voit sur la figure 1, les mutations sont réparties sur l'ensemble du gène mais clairement regroupées au sein des domaines présumés fonctionnels, surtout dans le premier domaine de fixation de l'ATP (NBF1) et dans le domaine NBF2. Quelques mutations ont été retrouvées dans le domaine $\mathrm{R}$, dont la fonction reste une énigme.

\section{Le rôle du CFTR}

L'obtention d'un ADNc complet et intègre, prérequis aux études d'expression, a d'emblée posé problème, les constructions initialement effectuées s'avérant d'une instabilité extrême. Les chercheurs ont finalement identifié la cause de leurs déboires : la séquence de l'exon $6 \mathrm{~B}$ contient un promoteur bactérien cryptique probablement responsable de la production d'une molécule toxique pour $E$. coli. C'est en inactivant ce promoteur par mutagenèse dirigée, sans modifier la séquence codante qui le contient, qu'il a été possible d'obtenir des recombinants stables permettant d'analyser l'expression de la protéine. Ayant ainsi levé l'obstacle, plusieurs groupes $[4,5]$ ont pu, indépendamment, démontrer que lorsque l'ADNc du gène CFTR normal était introduit dans des cellules épithéliales mutantes 


\section{Domaines}

\section{Les mutations du CFTR (juin 1991)}

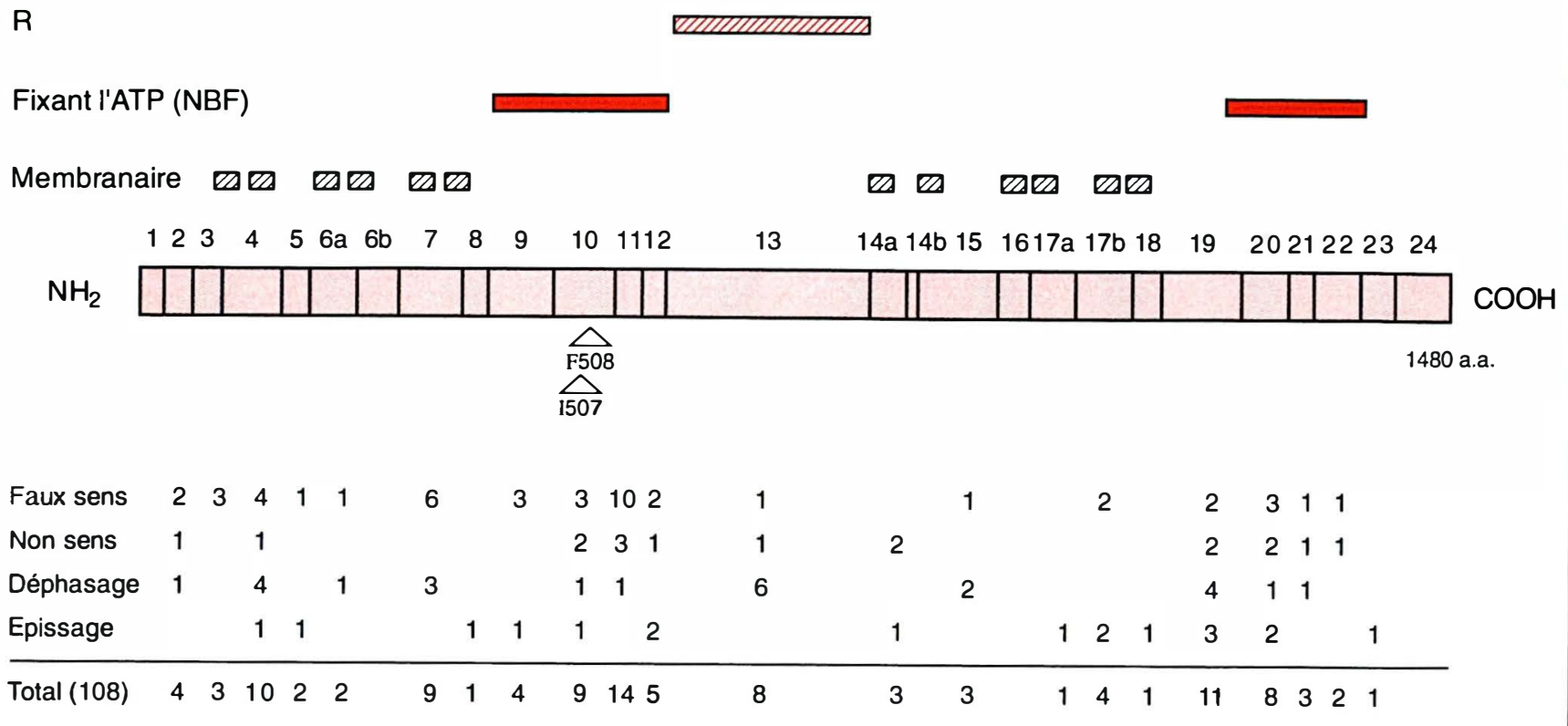

Figure 1. Les mutations de la protéine CFTR. Au-dessus des 27 exons du gène qui sont figurés et numérotés, la position des différents domaines protéiques est délimitée par des rectangles hachurés (zones hydrophobes, domaine $R$ ) ou ombrés rouges (NBF1 et NBF2 : nucleotide binding fold, zones de fixation de I'ATP). La partie inférieure de la figure présente la nature, le nombre et la localisation exonique des différentes mutations caractérisées par les différents laboratoires au sein du Consortium international (avril 1991).

provenant de malades homozygotes pour la mutation $\Delta F 508$, les cellules ainsi transfectées ne présentaient plus le phénotype mutant (le défaut d'activation des canaux $\mathrm{Cl}^{-}$contrôlée par l'AMPc). Cette restauration d'une sécrétion normale du chlore a confirmé le fait que la séquence clonée au cours de l'été 1989 est bien celle qui, mutée, est à l'origine de la mucoviscidose. Ces résultats, différés par les difficultés rencontrées dans la reconstruction de l'ADNc, ont permis d'aborder l'étude des relations entre la structure et la fonction du CFTR, et de confirmer par une série d'expériences in vitro que la protéine est membranaire, qu'elle peut être phosphorylée et qu'elle est normalement glycosylée, la glycosylation ne semblant pas nécessaire à l'activité biologique.

Mais quelle est la fonction du CFTR ? L'une des trois hypothèses avancées postule que la protéine CFTR est un canal $\mathrm{Cl}^{-}$. Mais elle pourrait tout aussi bien contrôler un canal $\mathrm{Cl}^{-}$auquel elle serait associée, ou exercer cette fonction de régulation par un effet indirect, en permettant le transport de fac- teurs inhibiteurs ou activateurs d'une activité de transport d'ions. Il s'avère qu'à l'inverse de la protéine mutante $\Delta$ F508 [6], la protéine CFTR normale peut être exprimée et fonctionner dans des cellules (HeLa, NIH3T3, CHO) qui normalement en sont dépourvues. Ces données invitent à spéculer que si la protéine CFTR n'est pas elle-même un canal $\mathrm{Cl}^{-}$, sa présence dans ces cellules pourrait permettre l'activation, par l'AMPc, d'hypothétiques canaux $\mathrm{Cl}^{-}$endogènes, cryptiques. A l'appui de ces hypothèses, il faut citer les résultats obtenus récemment de façon élégante par le groupe de Riordan [7] qui a montré que lorsque le CFTR est transfecté dans des cellules non épithéliales d'invertébré ( $\mathrm{Sf} 9$ ) via le système du baculovirus, il confere à ces cellules une perméabilité aux anions qui est stimulée par l'AMPc.

Ces observations suggèrent fortement que le CFTR est un canal $\mathrm{Cl}^{-}$contrôlé par l'AMPc. Des résultats récents, par exemple le fait d'observer que des mutations spécifiquement introduites dans les peptides insérés dans la membrane altèrent la sélectivité aux anions
[8], confortent cette présomption et rendent moins vraisemblables les autres hypothèses. Si le rôle des régions $N B F$, dont l'importance fonctionnelle est fortement suggérée par la densité des mutations naturelles identifiées en leur sein, reste quelque peu énigmatique, celui du domaine $\mathrm{R}$ commence à être mieux compris. Cette sous-unité contient quatre sérines dont la phosphorylation in vivo est nécessaire à l'ouverture du canal : en absence de phosphorylation, on n'observe pas de flux de chlore, mais si l'on délète le domaine $\mathrm{R}$, le flux ionique est important et échappe à toute régulation $[9,10]$. Il semble donc que la sous-unité $\mathrm{R}$ ouvre ou ferme le canal en réponse à l'action de protéine-kinases activées par l'AMP cyclique, et il est probable que les sous-unités fixant l'ATP ont aussi un rôle dans cette action.

Le fait que le CFTR ressemble à un transporteur mais fonctionne comme un canal laisse penser qu'il pourrait assumer les deux rôles, le substrat transporté restant à identifier. Cette protéine-canal fonctionne-t-elle sous 
forme d'un monomère unifonctionnel, ou sous celle d'un homodimère (ou hétérodimère) multifonctionnel ? La réponse à ces questions pourraient bien modifier certains de nos concepts actuels sur le fonctionnement des canaux et transporteurs.

L'analyse du mécanisme d'action des mutations a engendré des premiers résultats [11] qui semblent montrer que le CFTR mutant $\Delta$ F508 ne rejoint pas normalement, à l'issue de sa synthèse, son site d'action présumé dans la membrane, au pôle apical de la cellule : il s'accumule et serait dégradé dans le réticulum endoplasmique avant d'atteindre le Golgi où sa maturation est censée normalement se poursuivre. Cette anomalie du transit intracellulaire constituerait donc la lésion moléculaire principale de la mucoviscidose : la protéine est absente $\mathrm{du}$ lieu où son fonctionnement est requis. Il est probable, mais non vérifié, que des cellules hétérozygotes $\Delta$ F508 sont dépourvues, à leur surface, du CFTR anormal qui est retenu dans la cellule. En toute hypothèse, cette rétention intracellulaire d'un produit immature mais partiellement actif en un site aberrant pourrait être responsable d'effets délétères plus sévères que ceux résultant de son absence complète.

\section{Des orientations nouvelles pour la recherche thérapeutique}

L'analyse moléculaire et cellulaire de la fonction de la protéine CFTR devrait permettre, dès lors qu'il sera possible de la produire en quantité notable, la mise en œuvre d'approches pharmacologiques nouvelles. Les observations récentes peuvent même inciter à rechercher et tester des molécules capables de modifier la distribution et le transit intracellulaire des protéines. Parmi les systèmes d'expression potentiels, ceux qui utilisent la levure pourraient présenter un grand intérêt, si l'on se souvient qu'il existe dans cet organisme des protéines de la même famille (STE6), et compte tenu de la puissance des outils génétiques offerts à l'expérimentateur. Plusieurs laboratoires ont initié la création d'un modèle animal de la maladie en tentant d'inactiver, par recombinaison homologue, le gène CFTR murin. Les $\mathrm{m} / \mathrm{s} n^{\circ} 10$, vol. 7, décembre 91 souris transgéniques pourraient constituer un outil précieux pour inventorier les cibles tissulaires d'expression du gène, et établir par transimmortalisation les lignées cellulaires nécessaires aux études fonctionnelles et pharmacologiques. Enfin, des approches de thérapie génique, fondées sur la livraison d'un gène normal aux cellules épithéliales des voies aériennes supérieures, via un adénovirus recombinant ou à l'aide d'autres systèmes de transfert (protéoliposomes par exemple), sont actuellement considérées, et pour certaines initiées, s'appuyant sur les résultats obtenus chez l'animal sur le modèle $\alpha 1$-antitrypsine par M. Perricaudet, $\mathrm{R}$. Crystal, et leurs collabo-

\section{RÉFÉRENCES}

1. Goossens M. La découverte du gène de la mucoviscidose. médecine/sciences $1989 ; 5$ : 589-92.

2. Cuppens H, Marynen P, De Boeck C, et al. A child, homozygous for a stop codon in exon 11, shows milder cystic fibrosis symptoms than her heterozygous nephew. $J$ Med Genet 1990 ; 27 : 717-9.

3. Cutting $G$, Kash L, Rosenstein B, Tsui L, Kazazian H Jr, Antonarakis S. Two patients with cystic fibrosis, nonsense mutations in each cystic fibrosis gene, and mild pulmonary disease. $N$ Engl J Med 1990 ; 323 : 1685-9.

4. Rich D, Anderson M, Gregory R, et al. Expression of cystic fibrosis transmembrane conductance regulator corrects defective chloride channel regulation in cystic fibrosis airway epithelial cells. Nature $1990 ; 347$ : 358-63.

5. Drumm M, Pope H, Cliff W, et al. Correction of the cystic fibrosis defect in vitro by retrovirus-mediated gene transfert. Cell 1990 ; 62: 1227-33.

6. Anderson M, Rich D, Gregory R, Smith A, Welsh M. Generation of cAMPactivated chloride currents by expression of CFTR. Science 1991; 251 : 679-82.

7. Kartner N, Hanrahan J, Jensen $\mathrm{T}$, et al. Expression of the cystic fibrosis gene in nonepithelial invertebrate cells produces a regulated anion conductance. Cell 1991; 64 : 681-91.

8. Anderson MP, Gregory RJ, Thompson $\mathrm{S}$, et al. Demonstration that CFTR is a chloride channel by alteration of its anion selectivity. Science $1991 ; 253$ : 202-5.

9. Cheng SH, Rich DP, Marshall RJ, Gregory RJ, Welsh MJ, Smith AE. Phosphorylation of the R domain by cAMP-dependant proteine kinase regulates the CFTR chloride channel Cell 1991 ; $66 \cdot 1027-36$.

10. Rich DP, Gregory RJ, Anderson PM, Manavalan P, Smith AE, Welsh MJ. Effect of deleting the R domain on CFTR-generated chloride channel. Science $1991 ; 253$ : 205-7. 11. Cheng S, Gregory R, Marshall J, et al. sing of CFTR is the molecular basis of most cystic fibrosis. Cell $1990 ; 63$ : 827-34.

12. Kahn A. Les perspectives de thérapie génique de la mucoviscidose. médecine/sciences 1991 ; 7 : 84-5. rateurs de Transgène. Les perspectives de thérapie génique de la mucoviscidose ont été récemment évoquées dans cette revue [12].

Bien des obstacles restent encore à surmonter: la protéine reste difficile à produire en quantité suffisante, et la palette des anticorps spécifiques demeure pour l'instant trop restreinte. Le rôle du CFTR, son site d'action précis, les molécules avec lesquelles il interagit pour exercer sa fonction, sont autant d'éléments qui restent à élucider pour donner aux recherches thérapeutiques les appuis cohérents qui lui manquent encore

\section{Summary}

Biology of cystic fibrosis : recent advances and perspectives

Recent work has shown that the CFTR protcin acts like a cAMP. regulated chloride channel. More than a hundred mutations altering its function have been characterized in differents centers over the world. The most common CF mutation (the deletion of a phenylalanine at position 508 , or $\Delta \mathrm{F} 508$ ), which accounts for $70 \%$ of the global defective gene pool, is a $3 \mathrm{bp}$ deletion located in the first ATPbinding domain, and seems to cause a complete absence of the mature gene product. The other mutations are rare: some arc found mostly in population or ethnic groups, and most of them are clustered in the ATP-binding and transmembrane domains. They result in CF by impairing chloride secretion, but some, like the $\Delta \mathrm{F} 508$ defect, could also be deleterious to the cell metabolism. Some observations suggest that total absence of CFTR is not lethal, since patients with a presumably null CFTR phenotype can have a mild clinical course of $\mathrm{CF}$. It is possible that CFTR is a multifunctional protein, acting as a channel as well as a structure involved in the regulation of other cellular activities.

\section{TIRÉS A PART}

M. Goossens 